\title{
Géolinguistique
}

16 | 2016

Varia

\section{Vers un atlas de la langue de la mer en Dalmatie et Kvarner : le projet JAPRK}

Towards an Atlas of the Language of the Sea in Dalmatia and Kvarner: The JAPRK Project

Nikola Vuletić et Vladimir Skračić

\section{OpenEdition}

Journals

Édition électronique

URL : http://journals.openedition.org/geolinguistique/507

DOI : 10.4000/geolinguistique.507

ISSN : 2650-8176

Éditeur

UGA Éditions/Université Grenoble Alpes

Édition imprimée

Date de publication : 1 décembre 2016

Pagination : 91-105

ISBN : 978-2-84310-342-1

ISSN : 0761-9081

\section{Référence électronique}

Nikola Vuletić et Vladimir Skračić, «Vers un atlas de la langue de la mer en Dalmatie et Kvarner : le projet JAPRK », Géolinguistique [En ligne], 16 | 2016, mis en ligne le 15 février 2019, consulté le 10 novembre 2020. URL : http://journals.openedition.org/geolinguistique/507 ; DOI : https://doi.org/ 10.4000/geolinguistique.507 


\title{
Vers un atlas de la langue de la mer en Dalmatie et Kvarner : le projet JAPRK
}

\author{
Nikola Vuletić et Vladimir Skračić \\ Université de Zadar (Croatie)
}

\section{Résumé}

Ce travail est une réflexion sur les choix méthodologiques opérés dans l'élaboration et la mise en œuvre du projet de l'Atlas linguistique de la culture marine de la Dalmatie et du Kvarner (JAPRK). Ce projet est destiné à documenter la variation diatopique du lexique de la mer d'une bonne partie de l'Adriatique orientale et de le présenter sous forme d'un atlas linguistique et ethnographique. Nous mettrons en évidence les étapes de la réalisation du projet : la construction du questionnaire, les caractéristiques de l'espace et du réseau de l'enquête, la sélection de témoins, la menée d'enquêtes et le traitement des donnés. Afin d'illustrer les premiers résultats obtenus, on termine par une brève étude d'une carte d'essai.

\section{Mots-clés}

Atlas linguistique, Dalmatie, Kvarner, lexique de la mer.

\section{Abstract}

This paper brings some reflections on the methodological choices guiding the elaboration and the implementation of the project Linguistic Atlas of the Maritime Culture in Dalmatia and Kvarner. The aim of project is to document the diatopic variation of the maritime lexicon of the best part of the Eastern Adriatic region and to present it in the form of a linguistic and ethnographic atlas. We shall consider different stages of the realization of the project, such as the construction of the questionnaire, the characteristics of the space concerned and the network of survey locations, the 
selection of the informants, the course of the surveys and the data treatment. In order to illustrate the first results obtained, we conclude with a short study of one of preliminary maps.

\section{Keywords}

Linguistic atlas, Dalmatia, Kvarner, maritime lexicon.

\section{Présentation}

Toute entreprise géolinguistique visant à documenter la langue des marins et des pêcheurs, notamment dans le contexte d'un pays méditerranéen, s'inspire inévitablement des idées et de la méthodologie de cette entreprise monumentale qu'est l'Atlas linguistique méditerranéen (ALM), malheureusement arrêtée après la finalisation des enquêtes et la publication de quelques cartes d'essai (Cortelazzo, 1971). La liste des publications les plus importantes influencées par l'ALM inclut : la thèse de Berr (1973) sur la faune marine de la Bretagne, l'atlas linguistique (Le Berre \& Le Dû, 2008) élaboré à partir de celle-là ; le Léxico de los marineros peninsulares (Alvar, 1985-1989) ; l'étude monumentale de Vinja (1986) sur la faune des côtes croates et monténégrines; 1'Atlas linguístico do litoral português: fauna e flora de Vitorino (1987) ; l'Atlas léxico marinero de Asturias ALMA (Barriuso Fernández, 2002); le deuxième volume du Nouvel Atlas linguistique et ethnographique de la Corse - NALC (Dalbera-Stefanaggi \& Miniconi, 2008 [1999]); l'atlas plurilingue du vocabulaire maritime en Istrie - LAPTIG (Filipi \& Buršić Giudici, 2013) ${ }^{1}$.

À celles-ci s'ajoutent les projets géolinguistiques en cours de réalisation portant sur la langue de la mer, tels que le Vocabolario-atlante della cultura marinara in Sicilia (voir Ruffino \& D'Avenia, 2010) ou l'Atlas linguistique des côtes de l'Arc nord-occidental de la Méditerranée. Le reflet de cette tradition se trouve également dans notre projet d'Atlas linguistique de la culture marine de la Dalmatie et du Kvarner (en croate : Jezični atlas pomorske i ribarske kulture Dalmacije i Kvarnera - JAPRK), mis en chantier depuis le mois de janvier 2015. Il est en même temps un déploiement des expériences acquises lors des recherches toponymiques sur les îles de la Dalmatie septentrionale (voir Vuletić \& Skračić, 2016). Ces îles constituent la moitié des îles croates habitées (au nombre de 45 en ce moment).

1. Les matériaux publiés par Alvar (1985-1989), Vinja (1986) et Filipi et Buršić Giudici (2013) se présentent sous la forme de listes lexicales, sans cartes d'accompagnement. 
Dans un grand nombre de localités nous avons pu constater un effondrement démographique irréversible des communautés de pêcheurs autrefois florissantes. Tout un ensemble de pratiques traditionnelles de navigation et de pêche, lié à une manière de vivre la mer, est déjà passé à l'usage résiduel, et les effets linguistiques de ce processus irréversible ne sauraient manquer de se manifester. En partant de cette évidence, les objectifs principaux de notre projet sont :

- de créer un outil pour la connaissance de la variation du lexique traditionnel de la mer des variétés basilectales, à partir de relevés réalisés dans un nombre représentatif de localités côtières et insulaires de la Dalmatie et du golfe de Kvarner, présentés sous la forme des cartes linguistiques ${ }^{2}$;

- d'accompagner ces relevés des textes oraux illustrant les pratiques traditionnelles de navigation et de pêche ;

- et dans un second temps, de décrire la structure géolinguistique du lexique maritime croate du point de vue synchronique, et de sa formation historique, en accordant une attention particulière aux effets des contacts linguistiques, décisifs pour la survivance de nombreux éléments lexicaux romans (dalmates, vénitiens et, en général, italo-romans) et grecs dans les variétés croates ${ }^{3}$. À ceux-ci s'ajoute un objectif éducatif qui est celui d'inclure dans les activités de recherche les étudiants de l'université de Zadar provenant des localités du réseau d'enquête. Le projet jouit actuellement d'une subvention généreuse de l'université de Zadar.

\section{Un questionnaire pour la communauté}

Dans l'élaboration du questionnaire, nous sommes partis d'une expérience double avec le questionnaire de l'ALM concernant les côtes orientales de l'Adriatique : aux données de Deanović (1958, 1959, 1962, 1966, 1967), datant des années 1950 et 1960, s'ajoutent nos enquêtes, effectuées entre 2011 et 2014 dans les localités insulaires de la Dalmatie septentrionale. Il s'est révélé que ce questionnaire (composé de 810 questions onomasiologiques et 35 supplémentaires semi-ouvertes) contenait un nombre élevé de

2. Comme avait souligné Terracini (1964 : 5), un atlas linguistique n'est pas un objectif, mais un point de départ pour l'identification de phénomènes déterminés, au sein du système linguistique de la communauté.

3. Pour un aperçu de la stratification des éléments lexicaux romans et grecs dans les variétés croates, sur l'exemple du lexique maritime, voir p. ex. Deanović (1966-1967). 
notions peu pertinentes du point de vue de la culture maritime de l'Adriatique orientale.

D'autre part, nos témoins signalaient souvent l'absence de certains engins de pêche, de nombreuses pratiques, des traditions et des institutions sociales qu'ils jugeaient importantes. À partir de cette expérience, l'idée s'est imposée d'adopter une approche ascendante (bottom-up), en construisant un questionnaire propre à la communauté, sortie d'elle-même en quelque sorte. Ce questionnaire, par la suite, a été mis à la vérification auprès des pêcheurs de sept communautés insulaires (Banjol sur l'île de Rab ; Kali sur l'île d'Ugljan; Mali Iž sur l'île d'Iž ; Prvić Luka sur l'île de Prvić ; Kaprije, Murter et Zlarin sur les îles éponymes). Grâce à leurs commentaires et suggestions, nous avons abouti à un questionnaire de 540 questions onomasiologiques ${ }^{4}$.

La structure générale du questionnaire s'inspire directement de celle du deuxième volume du NALC. Les questions onomasiologiques s'y répartissent selon les thèmes suivants : le milieu physique (35); la météorologie (30) ; la navigation et le port (24) ; l'embarcation en bois (161) ; la vie du pêcheur (28); les engins de pêche (175); la faune et la flore marines (187). Le support du questionnaire est constitué, comme dans le cas de l'ALM et du $N A L C$, par un album avec les représentations d'animaux marins, de différents engins de pêche et de détails de construction des embarcations traditionnelles. Or, s'il est vrai que la technique d'élicitation directe au moyen d'un questionnaire, grâce à son caractère standardisé, assure un maximum de continuité entre les réponses de différentes localités (voir p. ex. Seiler, 2010 : 511-513), il est d'autant plus vrai que celle-ci présente un risque pour la fiabilité des relevés, puisque «la parole de l'enquête est sous la forte dépendance de celle de l'enquêteur» (Bres, 1999: 65).

En cherchant à atteindre un équilibre entre la demande d'efficacité de l'enquête et laisser l'initiative aux témoins, nous avons ajouté des questions ouvertes au questionnaire, devant contribuer à la spontanéité des entretiens et au versant ethnographique du projet. Ces dernières touchent la prévision météorologique traditionnelle, l'expérience de la navigation, la construction d'une embarcation traditionnelle en bois, un aperçu des pratiques de pêche en usage dans une localité donnée, les normes régissant la répartition du lot et le fonctionnement de la compagnie des pêcheurs, le processus de monter et de teindre un filet, la construction des nasses, etc.

4. La réduction du questionnaire de l'ALM a abouti à 436 questions dans le cas de l'atlas de Filipi et Buršić Giudici (2013), voire à 582 questions dans le Vocabolarioatlante della cultura marinara in Sicilia (Ruffino \& D'Avenia, 2010). 
Enfin, il est important de souligner que Vinja (1986) a déjà fait un inventaire exhaustif des noms vernaculaires des animaux marins, issus de ces enquêtes menées vers la fin des années 1950 dans 175 localités des côtes croates et monténégrines. Étant donné que le maillage de points pour lequel nous avons opté n'est pas si serré, la partie de notre questionnaire réservée à la faune marine n'a pas, bien évidemment, pour but de dépasser ses relevés, mais de les compléter.

\section{L'espace et le réseau d'enquête}

La zone intéressée par notre enquête comprend les côtes du golfe de Kvarner et celles de la Dalmatie. Le golfe de Kvarner est une mer intérieure de la mer Adriatique, située dans un triangle dont les côtes sont : la côte occidentale de l'Istrie, la côte continentale escarpée à l'est et les îles de la Dalmatie septentrionale au sud-est. Cette mer est parsemée de plus de 60 îles, dont les plus grandes sont Cres, Krk, Lošinj et Rab.

La perception contemporaine de la Dalmatie, dont l'étendue historique fut beaucoup plus large, coïncide avec celui de quatre comitats ou županije (unités administratives nationales), dont les centres sont les villes de la côte, à savoir : Zadar, Šibenik, Split et Dubrovnik ${ }^{5}$. En effet, la zone de notre enquête comprend presque toute la côte croate, à l'exception de la partie située le plus au nord-ouest (Istrie), qui a déjà fait l'objet d'un atlas du lexique maritime (Filipi \& Buršić Giudici, 2013).

La côte croate est extrêmement découpée, avec de nombreuses baies et pointes et un archipel de 1246 îles, îlots et écueils dressés devant la côte et la longeant du nord-ouest au sud-est, en plusieurs chapelets parallèles. Un ensemble de données numériques aide à saisir mentalement ses contours : tandis que la distance à vol d'oiseau entre le point le plus au nord-ouest (cap de Savudrija) et le plus au sud-est (cap de Prevlaka) est de $526 \mathrm{~km}$, la longueur totale de la ligne de rivage atteint $5835,3 \mathrm{~km}$, dont 1777,3 pour la côte et 4058 pour les îles.

Il est évident qu'un maillage d'enquête moyennement dense, qui tiendrait compte de la longueur totale du littoral croate et du nombre d'agglomérations, requerrait un nombre de points qui dépasserait largement nos capacités physiques, financières et logistiques. D'autre part, il faut prendre

5. Dit d'une autre manière, la Dalmatie moderne correspond à peu près au territoire du royaume de Dalmatie au sein de l'Empire austro-hongrois, exception faite de l'île de Rab, située dans la zone de Kvarner, et notamment des bouches de Kotor, rattachées au Monténégro depuis 1945 (voir Mirošević \& Faričić, 2011 : 124). 
en considération que la distance maritime - en prenant la route idéale entre deux points de l'Adriatique orientale coïncide, ou presque, avec la distance entre ces mêmes points effectuée à vol d'oiseau ${ }^{6}$. D'ailleurs, dans un espace maritime les distances ne sont souvent pas ce qu'elles peuvent sembler. Comme disaient Deanović et Folena (1959: 7-8) :

La continuità spaziale ha qui, com'è noto, un valore relativo: punti lontanissimi, legati da forti interessi e da intensi scambi, sono in realtà più vicini di punti contigui privi di stretti rapporti: spazio e tempo hanno sul mare una misura propria e diversa ${ }^{7}$.

Suite à ce qui a été dit, nous considérons qu'un réseau de 55 points (tableau 1 ; fig. 1), distribués le long d'une ligne idéale de $400 \mathrm{~km}$ environ (omission étant faite de l'Istrie, évidement), soit suffisamment serré pour assurer une représentation solide de la variation diatopique du lexique de la mer dans la zone intéressée. En établissant le réseau, nous avons aussi tenu compte de la réalité démographique de l'espace. Généralement, les îles croates ont subi une catastrophe démographique, notamment les petites îles habitées.

Les dernières décennies ont vu s'éteindre un certain nombre d'agglomérations de petites îles, telles Škarda, Srakane et d'autres encore sont en voie de disparition. La population insulaire croate ne fait que $3 \%$ de la population nationale. Le plus grave problème des îles est la moyenne d'âge très élevée de ses habitants (voisine de 60 ans) et par conséquent un taux d'activité très faible. Déjà dans son ensemble, les îles de l'archipel croate sont très petites et la population des deux tiers des petites îles habitées est extrêmement peu nombreuse (ne dépassant pas souvent cent habitants par île). D'autre part, l'île de Krk, à elle seule, compte presque 20000 habitants. Et pour terminer, seules 17 agglomérations des îles croates dépassent 1000 habitants. Tous ces facteurs et de nombreux autres ont été pris en considération lors du choix des critères pour les localités qu'il fallait soumettre à l'enquête. En voici certains d'entre eux :

Quasiment toutes les îles habitées sont représentées par au moins un point, exception étant faite pour certaines agglomérations insulaires de toutes petites îles à l'intérieur des grands archipels, tels que ceux de Zadar,

6. Ainsi la distance maritime entre deux points extrêmes de notre réseau, l'île d'Unije et le port de Cavtat, est de 220 milles marins ou d'environ $407 \mathrm{~km}$, tandis que la distance à vol d'oiseau entre les mêmes points est de $393 \mathrm{~km}$.

7. «La continuité spatiale a ici, comme il est notoire, une valeur relative : des points très éloignés, liés par intérêts forts et échanges intenses, sont en réalité plus proches entre eux que les points contigus dépourvus de rapports étroits : l'espace et le temps ont sur la mer une mesure propre et diverse.» 
de Šibenik et de Dubrovnik; deux points d'enquête sont réservés aux îles les plus grandes (avec une superficie supérieure à $\left.100 \mathrm{~km}^{2}\right)^{8}$.

Quant aux agglomérations de la côte, nous n'avons pas pu tenir compte du fait que la pêche artisanale se pratique presque partout. Nous n'avons sélectionné parmi ces localités que celles où l'importance économique de la pêche est comparable à l'importance qu'elle jouait, du point de vue historique, dans les localités insulaires, choisies comme points d'enquête.

Bien qu'il n'existe pas un seul point qui pourrait répondre à toutes les exigences et couvrir en même temps tous les champs du lexique marin, nous avons quand même cherché à ce que chaque point du réseau représente à la fois un centre relevant de la pêche et de la construction navale traditionnelle.

Tous les points croates de l'ALM ont été inclus dans le réseau du JAPRK, à l'exception de Mali Lošinj, qui figure parmi les points du LAPTIG (Filipi \& Buršić Giudici, 2013).

\begin{tabular}{|c|c|c|}
\hline \multirow[t]{2}{*}{ Kvarner (11) } & côte (3) & Bakar (01), Crikvenica (02), Senj (03) \\
\hline & îles (8) & $\begin{array}{l}\text { Klimno (04), Punat (05), Cres (06), } \\
\text { Martinšćica (07), Banjol (08), Unije (09), } \\
\text { Nerezine (10), Veli Lošinj (11) }\end{array}$ \\
\hline \multirow{2}{*}{$\begin{array}{l}\text { Dalmatie } \\
\text { septentrionale (22) }\end{array}$} & côte (3) & Privlaka (19), Novigrad (22), Rogoznica (34) \\
\hline & îles (19) & $\begin{array}{l}\text { Novalja (12), Pag (13), Premuda (14), } \\
\text { Silba (15), Olib (16), Ist (17), Molat (18), } \\
\text { Veli Rat (20), Sestrunj (21), Kali (23), } \\
\text { Mali Ǐz (24), Neviđane (25), Sali (26), } \\
\text { Vrgada (27), Betina (28), Murter-Kornati (29), } \\
\text { Prvić Luka (30), Zlarin (31), Kaprije (32), } \\
\text { Žrje (33) }\end{array}$ \\
\hline \multirow[t]{2}{*}{ Dalmatie centrale (11) } & côte (3) & Krilo Jesenice (36), Podgora (41), Brist (44) \\
\hline & îles (8) & $\begin{array}{l}\text { Trogir (36), Drvenik Veli (37), Grohote (38), } \\
\text { Milna (39), Sumartin (40), Stari Grad (42), } \\
\text { Vrboska (43), Komiža (45) }\end{array}$ \\
\hline \multirow[t]{2}{*}{ Dalmatie méridionale (10) } & côte (4) & $\begin{array}{l}\text { Trpanj (46), Kućište (47), Luka (50), } \\
\text { Cavtat (55) }\end{array}$ \\
\hline & îles (6) & $\begin{array}{l}\text { Vela Luka (48), Korčula (49), Lastovo (51), } \\
\text { Babino Polje-Sobra (52), Šipanska Luka (53), } \\
\text { Lopud (55) }\end{array}$ \\
\hline
\end{tabular}

Tableau 1. - Distribution des points d'enquête du JAPRK.

8. Parmi les îles dont la superficie dépasse $100 \mathrm{~km}^{2}$, les plus grandes sont Cres $\left(405,70 \mathrm{~km}^{2}\right)$ et $\mathrm{Krk}\left(405,22 \mathrm{~km}^{2}\right)$. 
Notre projet visant à recueillir le lexique de la mer des variétés basilectales autochtones, nous n'avons pas inséré dans le réseau d'enquête les grands ports urbains, comme ceux de Rijeka, de Zadar, de Split ou de Dubrovnik, puisqu'ils représentent une scène d'immigration, de nivellement linguistique et de formation de nouveaux dialectes urbains. Il faut avouer que dans toutes ces villes, centres de la pêche industrielle, la majorité des équipages est composée des marins-pêcheurs originaires des îles, qui, même quand ils résident en ville (ce qui n'est pas toujours le cas), maintiennent des rapports étroits avec leurs localités d'origine et par la suite contribuent aux répertoires linguistiques des centres urbains de leur résidence. Ces situations particulières requérant une méthodologie bien différente, l'étude du lexique de la mer des ports urbains reste à faire.

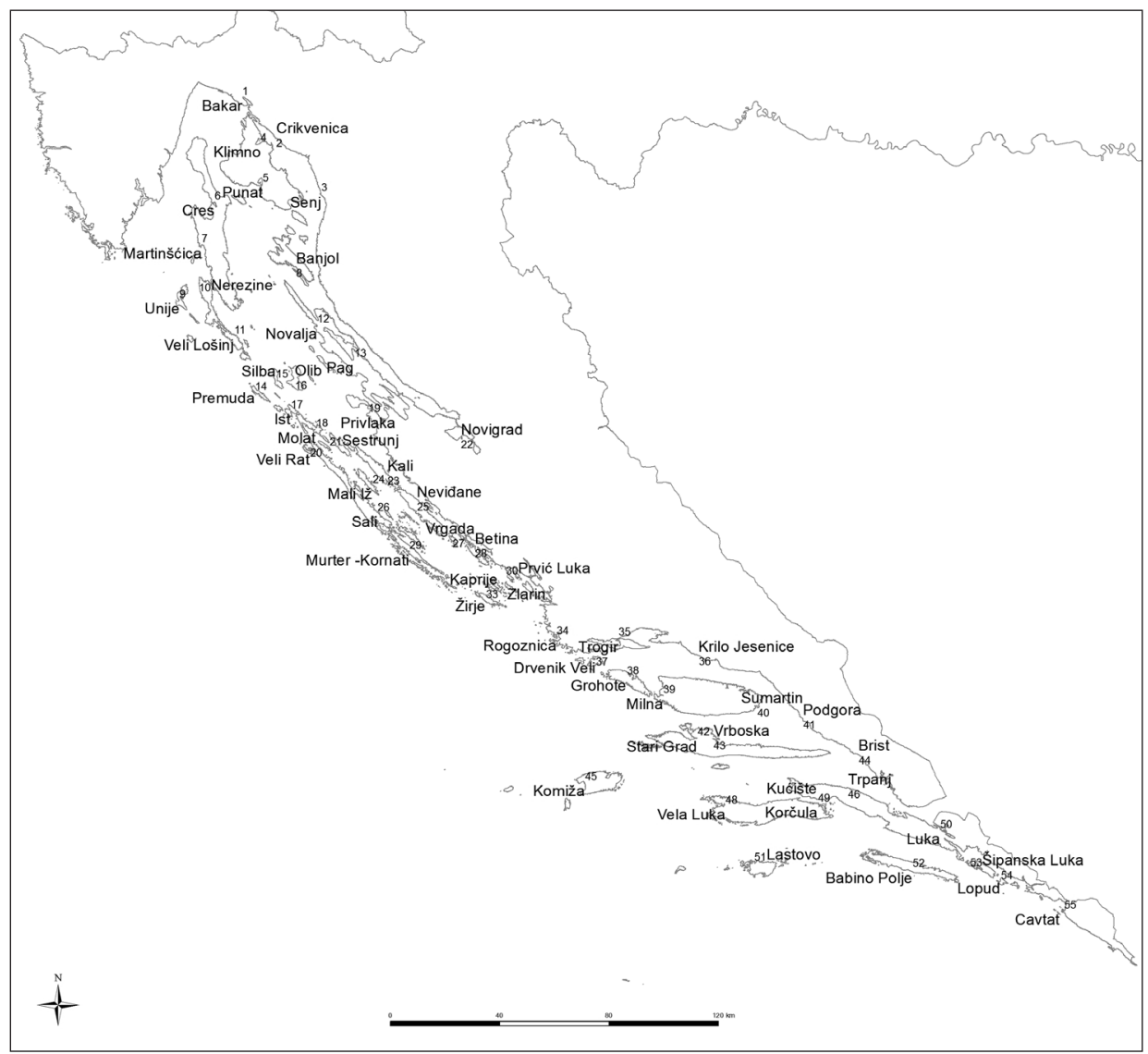

Figure 1. - Réseau d'enquête du JAPRK. 


\section{Le choix des témoins}

«C'est une langue de spécialité que l'on cherche à recueillir», soulignent les auteurs du deuxième volume du NALC dans leur introduction (DalberaStefanaggi \& Miniconi, 2008 [1999]). Cette réflexion est aussi d'une importance cruciale pour le discours sur les dimensions de la variation et les problèmes de la représentativité des témoins. Le lexique de la pêche traditionnelle est propre à un groupe sociolinguistique homogène : il s'agit d'hommes, généralement d'un degré d'instruction inférieur. Ensuite, les compagnies de pêcheurs de notre contexte sont traditionnellement composées de personnes venant d'une même localité, dont la population dépasse rarement 1000 habitants. Il s'agit de petites communautés, caractérisées par une situation linguistique relativement homogène, grâce surtout à un degré faible de la différenciation sociale (voir König, 2010 : 498-500). Le seul facteur socio-pragmatique susceptible de provoquer la variation reste l'âge, le facteur de sexe étant exclu dans un univers d'hommes qui est celui des pêcheurs.

Cependant, notre longue expérience des dialectologues travaillant sur les îles de la Dalmatie septentrionale a montré que, même dans le cas des îles qui jouissent de bonnes connexions avec les centres urbains de la côte, la différenciation diagénérationnelle joue un rôle mineur. Comme le dit Berruto (2010 : 227), il y a des espaces linguistiques où, grâce à la coaction des conditionnements d'ordre sociolinguistique, démographique, géographique et de circulation, la diatopie présente encore le facteur principal de la différenciation linguistique. Les îles croates, mais aussi certaines parties de la côte, répondent grosso modo à cette typologie.

Le choix des témoins est dicté, bien sûr, par les objectifs de l'enquête. Nous avons toujours cherché à les conformer au modèle d'un pêcheur expérimenté, originaire du lieu. Pour chacun des points nous avons interrogé au moins trois personnes, critère qui sera maintenu jusqu'à la dernière enquête. Dans certains cas, les témoins étaient plus de trois pour un certain nombre de localités. Leur âge moyen dépend des thèmes traités. Ainsi, pour faire ressortir de la mémoire le lexique de la voile ou de certaines techniques de pêche, on doit s'adresser aux personnes de plus de 60 ans, tandis que le lexique général de la pêche fait aussi partie des connaissances des témoins plus jeunes, âgés entre 40 et 60 ans. Arrivés aux maîtres charpentiers et calfats, il n'est plus question de choix : on cherche un expert local, quel que soit son âge ${ }^{9}$. Le degré d'instruction de nos témoins est

9. Sur l'île de Zlarin, le maître charpentier n'avait que 35 ans au moment de l'enquête. 
en principe secondaire, en prenant en considération qu'il était déjà très difficile de trouver ceux qui ne disposent que de l'instruction primaire. Évidemment, ce sont tous des hommes.

\section{Les enquêteurs et les enquêtes}

Dans la mesure du possible, nous avons opté pour le modèle de l'enquêteur dialectophone, en essayant de nous rapprocher au maximum de nos témoins. Nous avons donc veillé à diriger les entretiens en nous servant de la variété dialectale du lieu concerné ou au moins de la variété la plus proche de celle-ci. C'est une décision qui est issue de l'expérience des directeurs du projet. Les résultats obtenus à ce jour sont très satisfaisants : le témoin reconnaît dans le comportement linguistique du chercheur quelqu'un de semblable aux membres de sa communauté locale ou micro-régionale. Il se situe linguistiquement par rapport à l'enquêteur et l'entretien se déroule dans un climat de complicité et de confiance. Jusqu'à maintenant, les avantages tirés de cette approche ont largement dépassé le risque inhérent.

À côté des directeurs du projet, auteurs de cette contribution, qui ont réalisé les premières enquêtes en Dalmatie septentrionale, une équipe d'étudiants a été engagée depuis le début de l'année académique 2016-2017. Originaires principalement de la Dalmatie centrale et méridionale, ils se sont engagés à prendre en charge la direction des enquêtes dans leurs localités d'origine ou voisines, en remettant régulièrement les parties du questionnaire. C'est grâce à cette périodicité que la saisie et le contrôle des données sont plus efficaces et que nous pouvons présenter ici un cas particulièrement intéressant de la variation lexicale.

Pour ce qui est de la technique de l'enquête, chaque enquêteur est libre de s'adapter aux conditions qu'il trouve sur le terrain. La seule contrainte est d'enregistrer l'enquête sur un dictaphone digital et de remplir scrupuleusement le questionnaire. Les directeurs du projet ont toujours cherché à organiser l'entretien avec plusieurs témoins, en négligeant les précautions techniques (telles que le choix du lieu, pour éviter des bruits) au profit d'une situation dynamique, où les témoins s'inspirent mutuellement. Ici aussi, les avantages dépassent le risque : notre meilleur entretien fut celui de l'île de Zlarin, au beau milieu du chantier, avec une dizaine de pêcheurs parlant tous en même temps. Les témoins ont non seulement répondu à toutes les questions du questionnaire mais ils ont aussi tenu à signaler aux enquêteurs les divergences lexicales qui existent entre leur île et les îles voisines. À l'aide de deux dictaphones et avec deux enquêteurs, en notant en même temps les réponses directement sur le questionnaire, tout 
a été sauvé. Bien sûr, il a fallu saisir l'enquête dans le logiciel le plus tôt possible, en profitant de la mémoire fraîche que l'on avait d'un entretien si dynamique.

\section{Le traitement des données et la cartographie}

Suite à l'analyse phonétique et prosodique (aspect important en la matière de la dialectologie croate) des matériaux audio, les réponses des témoins à la partie onomasiologique du questionnaire sont saisies dans la base de données ${ }^{10}$. En vue d'une publication en libre accès et en pensant surtout à rendre service à nos témoins et à leurs communautés respectives, nous avons opté pour un triple système de transcription : à côte du système API et de celui de l'Atlas linguistique slave (OLA), un troisième type de transcription, basé sur l'alphabet croate (longue tradition dans les publications dialectologiques croates) est destiné au public non expert. La base de données est reliée au SIG (Système d'information géographique), ce qui rend possible la production automatisée de cartes linguistiques. On présentera des données brutes, sous la forme de cartes analytiques.

\section{Considérations finales avec l'analyse d'une carte d'essai}

Après avoir exposé, à grands traits, les lignes principales du projet, nous croyons utile de conclure ce travail de présentation par un exemple éloquent de la variation du lexique maritime en Dalmatie et au Kvarner. On a cartographié ici (fig. 2), d'une manière encore provisoire, les réponses à la question 343 - L'épuisette, obtenues dans l'ensemble des 55 points d'enquête. En continuation, les formes dialectales croates sont rendues dans la transcription API, avec la notation de la durée et la hauteur du ton de la voyelle tonique.

10. Les variétés croates de la zone étudiée se caractérisent par la présence de l'accent de hauteur (pitch accent). 


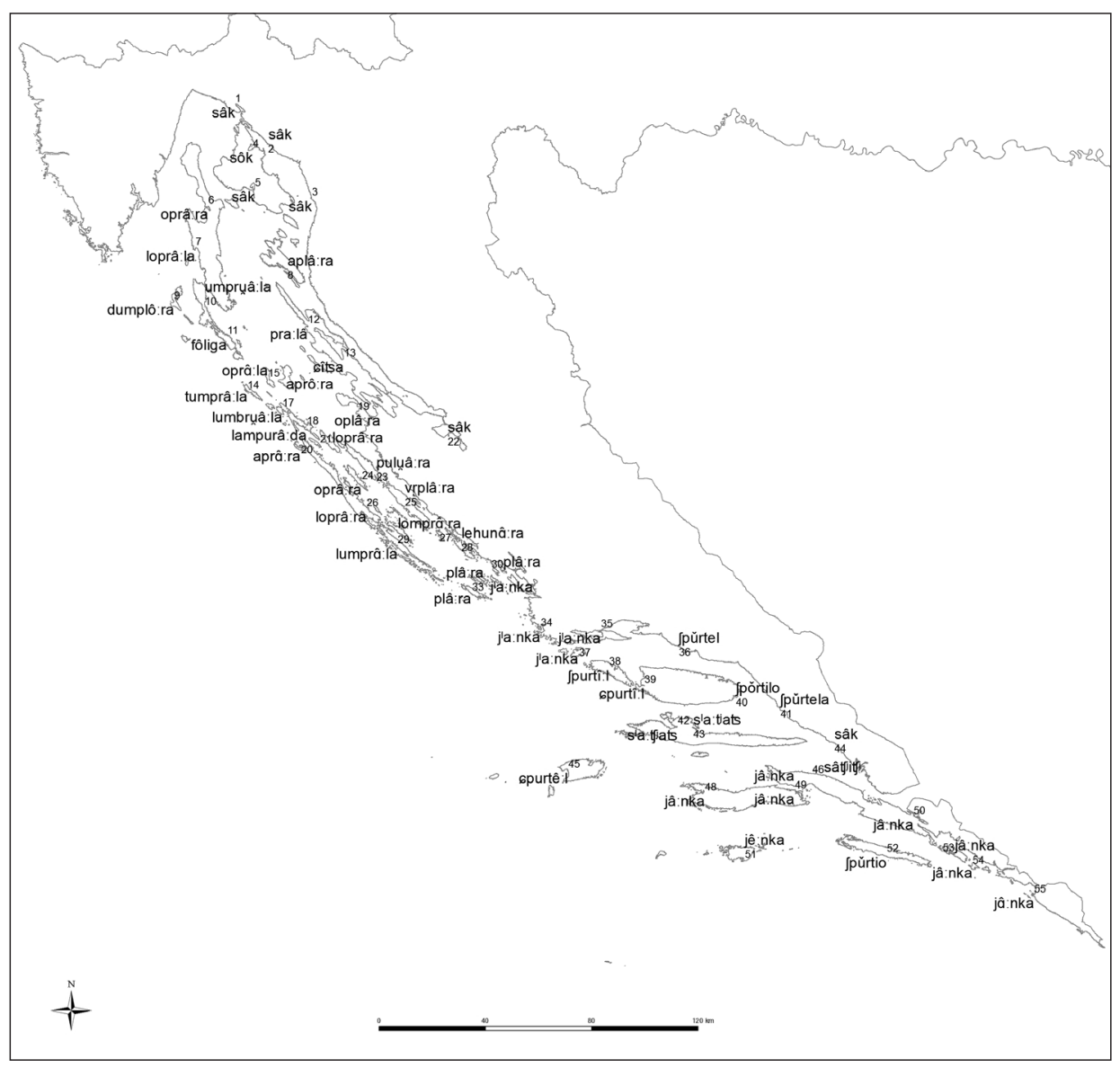

Figure 2. - Désignations de l'épuisette.

L'épuisette est un filet en forme de poche, monté sur un cerceau et fixé à un manche. Les pêcheurs de ces côtes s'en servaient surtout pour retirer le poisson d'un grand filet et le verser dans un sac. Nous avons identifié sept types lexicaux, remontant à un total de six bases étymologiques identifiées :

- Les reflets du lat. OPERARIA, sc. RETIS OPERARIA (JE, vol. II, s.v. lumprala), c'est-à-dire, du roman local (dit «dalmato-roman») *oprara. Ce type est propre à presque tous les points de la Dalmatie septentrionale, ainsi qu'aux îles de Kvarner, à l'exception de l'île de Krk. Les formes croates aboutissent à un grand nombre de variantes formelles : à partir de celles qui sont restées fidèles à la forme originaire (p. ex. [oprâ:ra], Cres), de celles avec l'article roman agglutiné (p. ex. [loprâ:ra], Sali), jusqu'à celles où différents changements 
phonétiques particuliers à différents dialectes, conjoints à l'action de l'étymologie populaire, ont rendu obscure la forme originaire sousjacente (p. ex. [dumplô:ra], Unije ; [lehuna^:ra], Betina ; [puluâa:ra], Kali ; [plâ:ra], Prvić Luka ; [vrplâ:ra], Neviđane).

- Les reflets de jamka ([jâ:nka] et variantes), forme dérivée du verbe jamiti «prendre» (ERHSJ, vol. I, s.v. jeti), occupent deux aires discontinues : la zone méridionale qui répond grosso modo au territoire de l'ancienne république de Dubrovnik, avec l'extension à l'île de Korčula, et la zone de transition entre la Dalmatie centrale et la Dalmatie septentrionale, ayant pour centre la ville de Trogir.

- Entre les deux aires du type janka s'insère une zone de reflets du vénitien sportela «petit cabas» (ERHSJ, vol. III, s.v. športel; JE, vol. III, s.v. spurćio), recouvrant la côte de la Dalmatie centrale et les îles de Šolta, Brač et Vis, avec un point isolé sur l'île de Mljet dans la zone méridionale. Les formes enregistrées sont surtout du genre masculin (p. ex. [Jpurtî:1], île de Šolta ; [Jpǒrtilo], Sumartin, sur l'île de Brač ; [Jpǔrtio], île de Mljet), avec quelques cas isolés du genre féminin (p. ex. [Jpǔrtela], Podgora). Le développement sémantique «cabas» $\rightarrow$ «épuisette» semble être propre aux variétés croates.

- Une zone compacte du type $s a k$, ancien latinisme (SACCU «sac») emprunté par toutes les langues slaves (ERHSJ, vol. III, s.v. sak), recouvre les côtes du golfe de Kvarner et l'île de Krk. Cependant, les reflets de sak se trouvent parsemés ici et là en Dalmatie (p. ex. [sâk] à Novigrad et Brist). Le type saćac, propre aux variétés de l'île de Hvar, représente à son tour une dérivation diminutive de sak.

- La forme [6îtsa], enregistrée uniquement à Pag (et dans le village voisin de Kolan), n'est pas claire.

- La forme [fôliga] à Veli Lošinj représente une incursion du type istro-vénitien volega (voir Filipi \& Buršić Giudici, 2013 : 199) dans la zone de reflets de *oprara.

Les désignations de l'épuisette illustre bien comment les Slaves, s'installant au Haut Moyen Âge sur les côtes de l'Adriatique orientale, se sont adaptés au nouvel entourage. Trois stratégies principales se dégagent : 1) l'emprunt lexical massif, sur le plan de la forme et du contenu, aux variétés romanes autochtones et, plus tard, de la même façon, au vénitien ; 2) le traitement créatif des formes empruntées, avec de nombreux changement formels et sémantiques ; 3) la néologie au sein du lexique slave héréditaire. Ces trois procédés relèvent de différentes dynamiques du contact slavo-roman qui, variant d'une zone de l'Adriatique à l'autre, et d'une 
époque à l'autre, prenait la forme d'un véritable plurilinguisme social ou d'un simple transfert culturel.

La variété des désignations sorties de ce processus séculaire dans un espace géographique si étroit n'est pas - nous sommes d'accord avec Deanović (1938) - un phénomène banal. Cette variété n'est pas seulement prononcée dans le champ ichthyonymique (voir p. ex. Vinja 1968), elle est également présente, comme nous venons de le voir, dans les autres champs du lexique de la mer. Dans l'espoir que notre atlas répondra tout d'abord aux rigoureuses exigences scientifiques, nous espérons aussi qu'il deviendra un instrument utile dans le cadre de la recherche du lexique méditerranéen. Il doit également répondre à la question de la place de l'Adriatique orientale dans le patrimoine linguistique de la mer. Et finalement, il est une reconnaissance à Skok, Vinja et Deanović, qui ont tellement fait pour préparer la voie de son développement.

\section{RÉFÉRENCES BIBLIOGRAPHIQUES}

Alvar Manuel, 1985-1989, Léxico de los marineros peninsulares, 4 vol., Madrid, Arco Libros.

Barriuso Fernández Emilio, 2002, Atlas léxico marinero de Asturias, Oviedo, Real Instituto de Estudios Asturianos/Gobierno del Principado de Asturias.

BerR Alan-Gwenog, 1973, Ichthyonymie bretonne, 3 vol., Brest, Université de Bretagne occidentale/Rennes, Institut armoricain de recherche.

Berruto Gaetano, 2010, «Identifying Dimensions of Linguistic Variation in a Language Space», dans P. Auer \& J. E. Schmidt (éds), Language and Space. An International Handbook of Linguistic Variation. Vol. 1 : Theories and Methods, Berlin, De Gruyter, p. 226-241.

Bres Jacques, 1999, «L'entretien et ses techniques », dans J.-L. Calvet et P. Dumont (éds), L'enquête sociolinguistique, Paris, L'Harmattan, p. 61-76.

Cortelazzo Michele, 1971, Atlante linguistico mediterraneo. Saggio delle carte, Florence, Fondazione Giorgio Cini \& Leo S. Olschki.

Dalbera-Stefanaggi Marie-José \& Miniconi Roger, 2008 [1999], Nouvel Atlas linguistique et ethnographique de la Corse. Vol. 2 : Le lexique de la mer, Ajaccio, Éditions Alain Piazzola/Paris, Éditions CTHS.

Deanović Mirko, 1938, «Divergences entre les emprunts latino-romans en Dalmatie (de Raguse à l'île de Šolta», Bulletin de la Société linguistique de Paris, vol. 39, nº 1, p. 25-48.

Deanović Mirko \& Folena Gianfranco, 1959, «Prospettive dell'Atlante linguistico mediterraneo », Bollettino dell'Atlante lingüístico mediterraneo, $\mathrm{n}^{\circ}$ 1, p. 7-12.

Deanović Mirko, 1958, «Terminologia marinara e peschereccia a Ragusavecchia (Cavtat)», Studia Romanica et Anglica Zagrabiensia, n 5, p. 3-22. 
Deanović Mirko, 1959, «Esperienze nell'Adriatico orientale con Questionario dell'ALM», Bollettino dell'Atlante lingüístico mediterraneo, $\mathrm{n}^{\circ}$ 1, p. 127-132.

DeAnović Mirko, 1962, «Lingvistički atlas Mediterana. Anketa u Boki Kotorskoj», Rad JAZU, n 327, p. 5-38.

Deanović Mirko, 1966, «Lingvistički atlas Mediterana II. Anketa na Visu (Komiža)», Rad JAZU, no 344, p. 5-35.

DeAnović Mirko, 1966-1967, «Stratificazione lessicale sulle coste orientali dell'Adriatico », Bollettino dell'Atlante linguistico mediterraneo, $\mathrm{n}^{\circ} 8-9$, p. 167-171.

DeAnović Mirko, 1967, «Lingvistički atlas Mediterana III. Anketa u Salima na Dugom Otoku », Rad JAZU, n 348, p. 27-60.

ERHSJ = Sкок Petar, 1971-1974, Etimologijski rječnik hrvatskoga ili srpskoga jezika, 4 vol., Zagreb, Jugoslavenska akdemija znanosti i umjetnosti.

FILIPI Goran \& BURŠIĆ GIUDICI Barbara, 2013, Lingvistički atlas pomorske terminologije istarskih govora, Zagreb, Dominović.

JE = VINJA Vojmir, 1998-2004, Jadranske etimologije: jadranske dopune Skokovu etimologijskom rječniku, 3 vol., Zagreb, Hrvatska akademija znanosti i umjestnosti / Školska knjiga.

KöNIG Werner, 2010, «Investigating Language in Space: Methods and Empirical Standards », dans P. Auer et J. E. Schmidt (éds), Language and Space. An International Handbook of Linguistic Variation. Vol. 1: Theories and Methods, Berlin, De Gruyter, p. 494-511.

Le BerRe Iwan \& Le Dû Jean (éds), 2009, Ichthyonymie bretonne, Brest, Université de Bretagne occidentale. DVD.

MirošEvić Lena \& FARIČIĆ Josip, 2011, «Perception of Dalmatia in Selected Foreign Lexicographic Publications », Geoadria, vol. 16, n 1, p. 119-140.

Ruffino Giovanni \& D'Avenia Elena 2010, Per un vocabolario-atlante della cultura marinara in Sicilia. Appunti e materiali, Palerme, Centro di studi filologici siciliani.

SEILER Günter, 2010, «Investing Language in Space: Questionnaire and Interview», dans P. Auer et J. E. Schmidt (éds), Language and Space. An International Handbook of Linguistic Variation. Vol. 1: Theories and Methods, Berlin, De Gruyter, p. 512-527.

Terracini Benvenuto, 1964, Saggio di un atlante linguistico della Sardegna, Zorino, Stamperia editoriale Rattero.

VINJA Vojmir, 1968, «Analyse du contenu des ichtyonymes. Les noms de Labrax lupus et de Chrysophrys aurata », Studia Romanica et Anglica Zagrabiensia, $\mathrm{n}^{\circ} 25-26$, p. 5-22.

VINJA Vojmir, 1986, Jadranska fauna: etimologija i struktura naziva, 2 vol., Zagreb, JAZU/Split, Logos.

Vitorino Gabriela 1987, Atlas linguístico do litoral português: fauna e flora, 2 vol., Lisbonne, Centro de Linguística da Universidade de Lisboa.

Vuletić Nikola \& SKračIĆ Vladimir, 2016, «El projecte del Centre d'Investigacions Onomàstiques Adriatiques de la Universitat de Zadar», Estudis Romànics, $\mathrm{n}^{\circ} 38$, p. 333-338. 